\title{
COMONOTONIC ADDITIVE OPERATORS AND THEIR REPRESENTATIONS
}

\author{
HEINZ J. SKALA \\ Hohefeld 18, 33100 Paderborn-Dahl, Germany
}

(Received 13 June, 1997)

\begin{abstract}
In a recent illuminating paper, June M. Parker [5] discussed Choquet integral representations of comonotonic additive functionals and related concepts. In our paper we provide a generalization of the Choquet integral and use this to obtain an integral representation for comonotonic additive operators.
\end{abstract}

1. Introduction. The present paper was motivated by the desire of the author to design a decision theory where the subjective measures of uncertainty (probabilities, capacities, or even only increasing mappings defined on some set of events) may vary with time. Instead of considering special function spaces it turned out that the main results could be proved for quite general Riesz spaces. The main technical tool is an "oldie" from the beginning of the theory of Riesz spaces, namely Freudenthal's spectral theorem.

Our paper may be considered as a follow-on to the one by June M. Parker [5]. All results in her interesting paper have Riesz space counterparts. As the arguments are easily transferred we do not discuss the details. Before reading this paper the interested reader is strongly recommended to consult [5] for additional motivation and further details. Concepts from the theory of Riesz spaces which are not explained in the present paper may be found in the excellent monograph by Luxemburg and Zaanen [4]. For the convenience of the reader we have completely adopted their notation. In addition, a few basic definitions and properties from the theory of Riesz spaces may be found in the appendix. Sometimes, for the sake of clarity, minor additions have been made.

2. The generalized Choquet integral. Let $L$ and $M$ be Riesz spaces and denote the set of all components of some nonzero $e \in L_{+}$by $C_{e}$. Recall that for any projection band $B$ we have $P_{B} e \in C_{e}$. Assume that the mapping $\varphi: C_{e} \rightarrow M$ has the properties

(i) $\varphi(0)=0$,

(ii) $\varphi\left(r_{1}\right) \geq \varphi\left(r_{2}\right)$ if $r_{1} \geq r_{2} ; r_{1}, r_{2} \in C_{e}$.

As in the real valued case it is easily verified that every $e$-step element $f$ has a "layered" representation $f=\sum_{k=1}^{n} \beta_{k} r_{k} ; \beta_{k} \in \mathbb{R} ; r_{1} \geq \ldots \geq r_{n} ; r_{k} \in C_{e}$ and it makes sense to define

$$
\Phi_{\varphi}(f)=C \int f d \varphi=\sum_{k=1}^{n} \beta_{k} \varphi\left(r_{k}\right), \beta_{k} \geq 0 ; r_{1} \geq \ldots \geq r_{n} ; r_{k} \in C_{e} .
$$

This operator may well be called generalized Choquet integral. As $\Phi_{\varphi}$ behaves additively on the partial sums of layered representations it follows that $\Phi_{\varphi}(f)$ is independent of the chosen layered representations of $f$. 
In order to define the generalized Choquet integral for more general elements of $L$ we assume that $L$ has the principal projection property whence Freudenthal's spectral theorem applies. (See [4, p. 247 ff.].)

Let $0 \leq f \leq(b-c) e$ for some nonzero $e \in L_{+} ; b, c \in \mathbb{R}_{+}$and assume that $\varphi:\left\{{ }_{e}^{f} r_{\alpha}: \alpha \in \mathbb{R}_{+}\right\} \rightarrow M_{+}$satisfies (i) and (ii); remember that the ${ }_{e}{ }_{e} r_{\alpha}$ are components of $e$ and ${ }_{e}^{f} r_{\alpha}={ }_{e}^{f} R_{\alpha} e=e-{ }_{e}^{f} p_{\alpha}$. (The set $\left\{{ }_{e}^{f} p_{\alpha}: \alpha \in \mathbb{R}_{+}\right\}$is usually called the spectral system of $f$ with respect to $e$.) It is natural to define lower and upper sums by

$$
\underline{S}(\pi, f, \varphi)=\sum_{k=1}^{n} \beta_{k} \varphi\left({ }_{e}^{f} r_{\alpha_{k}}\right) \text { and } \bar{S}(\pi, f, \varphi)=\sum_{k=1}^{n} \beta_{k} \varphi\left({ }_{e}^{f} r_{\alpha_{k-1}}\right)
$$

where $\beta_{k}:=\left(\alpha_{k}-\alpha_{k-1}\right)$ for any partition $\pi, 0=\alpha_{o}<\ldots<\alpha_{n}=b$. Evidently, for refining partitions, $\underline{S}(\pi, f, \varphi)$ is increasing and $\bar{S}(\pi, f, \varphi)$ is decreasing.

Definition 2.1. Let $L$ and $M$ be Riesz spaces, and assume that $L$ has the principal projection property. Let $f \in L_{+}$and suppose that $\varphi$ is defined on the complementary components of the spectral system of $f$ with respect to $e$ (i.e. $\varphi:\left\{{ }_{e}^{f} r_{\alpha}: \alpha \in \mathbb{R}_{+}\right\} \rightarrow M_{+}$) and satisfies (i) and (ii). The element $q \in M$ is said to be the generalized Choquet integral of $f \in L_{+}$with respect to $\varphi$ and we write $q=C \int f d \varphi$ if, for some $b, c \in \mathbb{R}_{+}$and $e \in L_{+}$, we have $0 \leq f \leq(b-c) e$ and to each $\varepsilon \in \mathbb{R}_{+}$there exists a $\delta \in \mathbb{R}_{+}$such that for any partition of $[0, b]$ with $|\pi| \leq \delta$ we have $q-\underline{S}(\pi, f, \varphi) \leq \varepsilon \varphi(e)$ and $\bar{S}(\pi, f, \varphi)-\varphi \leq \varepsilon \varphi(e)$, where $|\pi|$ denotes the maximal subinterval length in the partition $\pi$.

In other words, our definition demands that $q$ is the $\varphi(e)$-uniform limit of $\underline{S}(\pi, f, \varphi)$ and $\bar{S}(\pi, f, \varphi)$ respectively as $|\pi| \rightarrow 0$.

In general the integral just defined need neither exist nor be unique. Therefore we assume from now on that $M$ is Archimedean. This makes the integral unique, if it exists (cf. [4, p. 252]). Moreover we assume that for every $e \in L_{+}$, any $e$-uniform Cauchy sequence has an $e$-uniform limit, i.e. $M$ is uniformly complete. Observe that the class of uniformly complete Archimedean Riesz spaces is sufficiently large for almost all conceivable applications; e.g. every Dedekind $\sigma$-complete Riesz space is Archimedean and uniformly complete. For more information see [4, p. 276 ff.].

Definition 2.2. Two elements $f, g, \in L$ are called comonotonic if the set $\left\{{ }_{e} r_{\alpha},{ }_{e}^{g} r_{\alpha}: \alpha \in \mathbb{R}\right\}$ forms a chain.

THEOREM 2.3. Let L be a Riesz space possessing the principal projection property, and let $e$ be a fixed nonzero element in $L_{+}$. Denote by $I_{e}$ the ideal generated by $e$. Assume that $\varphi$ takes values in a uniformly complete Archimedean Riesz space M. If $\varphi$ is defined for all ${ }_{e}^{f} r_{\alpha} ; f \in I_{e} \cap L_{+}, \alpha \in \mathbb{R}_{+}$and satisfies (i) and (ii), then we can define an operator $\Phi_{\varphi}: I_{e} \cap L_{+} \rightarrow M$ by

$$
\Phi_{\varphi}(f)=C \int f d \varphi .
$$

$\Phi_{\varphi}(\cdot)$ is positive homogeneous increasing and additive for comonotonic elements.

Proof. If the operator $\Phi_{\varphi}$ exists, then it is trivially positive homogeneous and increasing. To prove its existence, let $0 \leq f \leq(b-c) e ; b, c \in \mathbb{R}_{+}, e \in L_{+}$. For any 
partition $\pi$ of $[0, b], 0=\alpha_{o}<\alpha_{1}<\ldots<\alpha_{n}=b$ with maximal subinterval length $|\pi|$ we have

$$
\bar{S}(\pi, f, \varphi)-\underline{S}(\pi, f, \varphi) \leq|\pi| \sum_{k=1}^{n}\left(\varphi\left({ }_{e}^{f} r_{\alpha_{k-1}}\right)-\varphi\left({ }_{e}^{f} r_{\alpha_{k}}\right)\right)=|\pi|\left(\varphi\left({ }_{e}^{f} r_{\alpha_{o}}\right)-\varphi\left({ }_{e}^{f} r_{\alpha_{n}}\right)\right)=|\pi| \varphi(e) .
$$

The last two equalities follow from the fact that $\varphi$ is increasing and the observations $r_{o}=e, r_{\alpha_{n}}=0$. This implies that for refining partitions $\pi_{m}$ with $\left|\pi_{m}\right| \rightarrow 0$ the sequence $\underline{S}\left(\pi_{m}, f, \varphi\right)$ (as well as $\left.\bar{S}\left(\pi_{m}, f, \varphi\right)\right)$ is $e$-uniform Cauchy. The assumptions on $M$ ensure that the limit exists and is unique, whence $C \int f d \varphi$ exists for any $f \in I_{e}$.

To verify that $\Phi_{\varphi}$ is additive for comonotonic elements $f, g$ assume $0 \leq f+g \leq(b-c) e$ for some $b, c \in \mathbb{R}_{+}, e \in L_{+}$. Then for any partition $0=\alpha_{o}<\alpha_{1}<\ldots<\alpha_{n}=b$ the set $\left\{{ }_{e}^{f} r_{\alpha_{0}}, \ldots,{ }_{e} r_{\alpha_{n}},{ }_{e}^{g} r_{\alpha_{0}}, \ldots,{ }_{e}^{g} r_{\alpha_{n}},{ }_{e}^{f+g} r_{\alpha_{0}}, \ldots,{ }_{e}^{f+g} r_{\alpha_{n}}\right\}$ forms a chain and we have

$$
\begin{aligned}
\underline{s}(\pi, f)+\underline{s}(\pi, g) & \leq \sum_{k=1}^{n}\left({ }_{e}^{f} R_{\alpha_{k-1}}-{ }_{e}^{f} R_{\alpha_{k}}\right) f+\sum_{k=1}^{n}\left({ }_{e}^{g} R_{\alpha_{k-1}}-{ }_{e}^{g} R_{\alpha_{k}}\right) g=f+g \\
& =\sum_{k=1}^{n}\left({ }_{e}^{f+g} R_{\alpha_{k-1}}-{ }_{e}^{f+g} R_{\alpha_{k}}\right)(f+g) \\
& \leq \underline{s}(\pi, f+g)+|\pi| e \leq \underline{s}(\pi, f)+\underline{s}(\pi, g)+3|\pi| e .
\end{aligned}
$$

Therefore $\underline{S}(\pi, f, \varphi)+\underline{S}(\pi, g, \varphi) \leq \underline{S}(\pi, f+g, \varphi)+|\pi| \varphi(e) \leq \underline{S}(\pi, f, \varphi)+\underline{S}(\pi, g, \varphi)+$ $3|\pi| \varphi(e)$. The existence and uniqueness of the limits for $|\pi| \rightarrow 0$ now ensure the comonotonic additivity of $\Phi_{\varphi}$.

REMARK. Obviously, for unbounded elements arguments via $e$-uniform approximation no longer hold. However, a spectral theorem for the unbounded case can still be obtained in the sense of order convergence (cf. [4, p. $258 \mathrm{ff}$.]). the corresponding generalization of Theorem 2.3. is easy to obtain.

3. Integral representations of comonotone additive operators. Let $L$ and $M$ be Riesz spaces. We are now interested in operators $\Phi: L \rightarrow M$ which are positive homogeneous, increasing and comonotonic additive, i.e. they satisfy

(i) $\Phi(\alpha f)=\alpha \Phi(f) ; \alpha \in \mathbb{R}_{+}, f \in L$,

(ii) $\Phi(f) \geq \Phi(g)$ if $f \geq g ; f, g \in L$,

(iii) $\Phi(f+g)=\Phi(f)+\Phi(g)$ if $f$ and $g$ are comonotone.

Obviously, for any nonzero $e \in L_{+}$the restriction of $\Phi$ to $C_{e}$ induces a mapping $\varphi_{\Phi}: C_{e} \rightarrow M$ on the components of $e$ which is nonnegative and increasing. This suggests the possibility to use Freudenthal's spectral theorem to obtain an integral representation theorem similar to the one given by Greco [3] and Schmeidler [6] for the real case. More general results obtained by different tools may be found in a forthcoming paper by Skala [7].

Theorem 3.1. Let $L$ and $M$ be two Riesz spaces where $L$ has the principal projection property and $M$ is Archimedean and uniformly complete. Let e be a nonzero element in $L_{+}$and let $\Phi: L \rightarrow M$ have properties (i), (ii) and (iii). For any nonnegative $f$ in the ideal $I_{e}$ generated by e the following holds: 


$$
\Phi(f)=C \int f d \varphi_{\Phi}, f \in I_{e} \cap L_{+} .
$$

Proof. Properties (i) and (ii) of $\Phi$ trivially imply that $\Phi$ is nonnegative, whence $\varphi_{\Phi}: C_{e} \rightarrow M$ is nonnegative and vanishes on the zero element. As $\Phi$ is increasing so is $\varphi_{\Phi} . f \in I_{e} \cap L_{+}$implies that there are $b, c \in \mathbb{R}_{+}$such that $0 \leq f \leq(b-c) e$. For any partition $\pi, 0=\alpha_{0}<\alpha_{1}<\ldots<\alpha_{n}=b$, define upper and lower sums as before, i.e.

$$
\underline{s}(\pi, f)=\sum_{k=1}^{n} \beta_{k_{e}^{f}} r_{\alpha_{k}} \text { and } \bar{s}(\pi, f)=\sum_{k=1}^{n} \beta_{k_{e}^{f}} r_{\alpha_{k-1}} ; \beta_{k}=\left(\alpha_{k}-\alpha_{k-1}\right) .
$$

By Freudenthal's spectral theorem $\underline{s}\left(\pi_{m}, f\right) \uparrow f$ and $\bar{s}\left(\pi_{m}, f\right) \downarrow f$ hold $e$-uniformly for refining partitions $\pi_{m},\left|\pi_{m}\right| \rightarrow 0$.

Observe that the ${ }_{e}^{f} r_{\alpha_{k}}, k=1, \ldots, n$ form a chain and belong to $C_{e}$. Hence

$$
\Phi(\underline{s}(\pi, f))=\sum_{k=1}^{n} \beta_{k} \varphi_{\Phi}\left({ }_{e}^{f} r_{\alpha_{k}}\right)=\underline{S}\left(\pi, f, \varphi_{\Phi}\right)
$$

and

$$
\Phi(\bar{s}(\pi, f))=\sum_{k=1}^{n} \beta_{k} \varphi_{\Phi}\left({ }_{e}^{f} r_{\alpha_{k-1}}\right)=\bar{S}\left(\pi, f, \varphi_{\Phi}\right)
$$

by the comonotonic additivity of $\Phi$. As $\Phi$ is increasing, $\Phi(\underline{s}(\pi, f)) \leq$ $\Phi(f) \leq \Phi(\bar{s}(\pi, f)) \quad$ and $\quad \Phi(\bar{s}(\pi, f))-\Phi(\underline{s}(\pi, f)) \leq|\pi| \varphi_{\Phi}\left(r_{0}\right)=|\pi| \varphi_{\Phi}(e)$. Thus $\Phi\left(\underline{s}\left(\pi_{m}, f\right)\right) \uparrow \Phi(f)$ and $\Phi\left(\bar{s}\left(\pi_{m}, f\right)\right) \downarrow \Phi(f)$ hold $\varphi_{\Phi}(e)$-uniformly for refining partitions $\pi_{m},\left|\pi_{m}\right| \rightarrow 0$. By the assumptions on $M$ this limit exists in $M$ and is unique.

REMARK. For unbounded $f$ we can no longer expect that $f$ is the $e$-uniform limit of both $\underline{s}\left(\pi_{m}, f\right)$ and $\bar{s}\left(\pi_{m}, f\right)$. However, a one sided approximation result is still possible if we replace $e$-uniform convergence by order convergence.

As an immediate consequence we essentially obtain Greco's [3] version of Shmeidler's [6] representation theorem.

COROllary 3.2. Suppose that IF is a $\sigma$-algebra of subsets of some set $X$ and denote by $L^{\infty}(X, I F)$ the sup-norm closure of the finite step functions. Each functional $\Phi: L_{+}^{\infty}(X, I F) \rightarrow \mathbb{R}_{+}$which is positively homogeneous, increasing, and comonotonic additive has an integral representation, i.e.

$$
\Phi(f)=C \int f d \varphi_{\Phi}:=R \int_{0}^{\sup f} \Phi\left(1_{\{f \geq \alpha\}}\right) d \alpha, f \in L_{+}^{\infty}(X, I F),
$$

where $R \int$ denotes the Riemann integral.

Proof. It is well known that $L^{\infty}(X, I F)$ is Dedekind $\sigma$-complete for any $\sigma$-algebra $I F$, and therefore Freudenthal's spectral theorem applies. Take the constant 1 function as $e$ and observe that the components of $e$ are just the characteristic functions $1_{A}$, $A \in I F$. Then $\varphi_{\Phi}(A)=\Phi\left(1_{A}\right), A \in I F$. 
4. The subadditivity theorem. Let $L$ be a Riesz space possessing the principal projection property. Assume that for some nonzero $e \in L_{+}$the mapping $\varphi: C_{e} \rightarrow M_{+}$takes values in a uniformly complete Archimedean Riesz space $M$ and satisfies (i) and (ii). It is an easy exercise to verify that if $C \int(f+g) d \varphi \leq C \int f d \varphi$ $+C \int g d \varphi, f, g \in I_{e} \cap L_{+}$, then $\varphi$ must be submodular on $C_{e}$. The converse is slightly more difficult to prove. The real valued version of this result dates back to Choquet [1]. For a detailed history and additional references the reader should consult Denneberg [2].

THEOREM 4.1. Let L be a Riesz space possessing the principal projection property and let $e \in L_{+}$be some nonzero element. Assume that the Riesz space $M$ is Archimedean and uniformly complete and that $\varphi: C_{e} \rightarrow M$ has the following properties:

(i) $\varphi(0)=0$,

(ii) $\varphi\left(r_{1}\right) \geq \varphi\left(r_{2}\right)$ if $r_{1} \geq r_{2} ; r_{1}, r_{2} \in C_{e}$,

(iii) $\varphi\left(r_{1} \vee r_{2}\right)+\varphi\left(r_{1} \wedge r_{2}\right) \leq \varphi\left(r_{1}\right)+\varphi\left(r_{2}\right) ; r_{1}, r_{2} \in C_{e}$.

Then

$$
C \int(f+g) d \varphi \leq C \int f d \varphi+C \int g d \varphi \text { for all } f, g \in I_{e} \cap I_{+} .
$$

Proof. Let $f, g \in I_{e} \cap I_{+}$. By assumption there exist $b, c \in \mathbb{R}_{+}$such that $0 \leq f+g \leq(b-c) e$. Freudenthal's spectral theorem ensures that

$$
S\left(\pi_{m}, f\right) \uparrow f \text { and } S\left(\pi_{m}, g\right) \uparrow g
$$

hold $e$-uniformly for any sequence of refining partitions $\pi_{m}$ with $\left|\pi_{m}\right| \rightarrow 0$. We may restrict the points of the partitions to the rational numbers (cf. also [4, p. 261]). Therefore, by the properties of $C \int(\cdot)$, it suffices to prove $C \int(f+g) d \varphi \leq$ $C \int f \mathrm{~d} \varphi+C \int g \mathrm{~d} \varphi$ for elements of the "layered" type

$$
f=\sum_{i=1}^{m}{ }_{e}^{f} r_{i}, g=\sum_{i=1}^{m}{ }_{e}^{g} r_{i} \text {, where }{ }_{e}^{f} r_{i},{ }_{e}^{g} r_{i} \in C_{e} .
$$

In the first step assume $f=\sum_{i=1}^{m}{ }_{e} r_{i}$ and $g={ }_{e}^{g} r_{1}$. We remember that $C_{e}$ is a Boolean algebra. By the submodularity of $\varphi$ we get

$$
\varphi\left({ }_{e}^{f} r_{1} \vee{ }_{e}^{g} r_{1}\right)+\varphi\left({ }_{e}^{f} r_{1} \wedge{ }_{e}^{g} r_{1}\right) \leq\left({ }_{e}^{f} r_{1}\right)+\varphi\left({ }_{e}^{g} r_{1}\right) .
$$

As ${ }_{e}^{f_{i}} r_{i} \geq{ }_{e}^{f} r_{i+1}$ obviously ${ }_{e}^{f} r_{i+1} \wedge\left({ }_{e}^{f} r_{i} \wedge{ }_{e}^{g} r_{1}\right)={ }_{e}^{f} r_{i+1} \wedge{ }_{e}^{g} r_{1}$ holds. Hence again by the submodularity of $\varphi$,

$$
\varphi\left({ }_{e}^{f} r_{i+1} \vee\left({ }_{e}^{f} r_{i} \wedge{ }_{e}^{g} r_{1}\right)\right)+\varphi\left({ }_{e}^{f} r_{i+1} \wedge{ }_{e}^{g} r_{1}\right) \leq \varphi\left({ }_{e}^{f} r_{i+1}\right)+\varphi\left({ }_{e}^{f} r_{i} \wedge{ }_{e}^{g} r_{1}\right) .
$$

Observing that ${ }_{e}^{f} r_{m+1}=0$, the summation of the inequalities results in

$$
C \int\left(f+{ }_{e}^{g} r_{1}\right) d \varphi \leq C \int f d \varphi+C \int{ }_{e}^{g} r_{1} d \varphi .
$$

Repeating this argument for ${ }_{e}^{g} r_{i}, i \geq 2$, finally gives the desired result. 
5. Appendix. In order to make the paper more self contained we give here a brief survey of some basic definitions and properties from the theory of Riesz spaces. For more details the reader is urged to consult the monograph by Luxemburg and Zaanen [4].

Definition 5.1. A Riesz space is an ordered vector space $L$ with the property that for any pair $f, g \in L$ their infimum and supremum exists in $L$. Obviously, every function space with the pointwise ordering is a Riesz space.

Definition 5.2. We call an element $f \in L_{+}$a component of $e \in L_{+}$if $f \wedge(e-f)=0$.

Definition 5.3. The band $B_{f}$ generated by a single element $f \in L$ is called a principal band. $L$ is said to have the principal projection property if every principal band in $L$ is a projection band.

The following implications were proved in [4].

$\begin{aligned} \text { Dedekind complete } & \rightarrow \text { Dedekind } \sigma \text {-complete } \rightarrow \text { projection property } \rightarrow \text { principal projection property } \rightarrow \text { Archimedean. }\end{aligned}$

Definition 5.4. Let $f \in L$ and $e \in L_{+}$be given. For any real $\alpha$, the component of $e$ in the principal projection band generated by $(\alpha e-f)^{+}$is denoted by ${ }_{e}^{f} p_{\alpha}$, or $p_{\alpha}$ in short, and the system $\left\{{ }_{e}^{f} p_{\alpha}: \alpha \in \mathbb{R}\right\}$ is called the spectral system of $f$ with respect to $e$.

Freudenthal's SPECTRAL THEOREM Let L be a Riesz space with the principal projection property and let $0 \neq e \in L_{+}$. For any $f$ in the principal ideal $I_{e}$ generated by e there exist sequences $\left(\underline{s}_{m}\right)_{m \in \mathbb{N}}$ and $\left(\bar{s}_{m}\right)_{m \in \mathbb{N}}$ of e-step elements such that $\underline{s}_{m} \uparrow f$ and $\bar{s}_{m} \downarrow f$ hold e-uniformly. Replacing e-uniform convergence by order convergence there exists a one sided generalization for any $f \in B_{e}$.

ACKnOwledgement. The author is grateful to an anonymous referee for proposals enhancing the readability and self containedness of the paper.

\section{REFERENCES}

1. G. Choquet, Theory of capacities, Annales de l'Institut Fourier 5 (1953/54), 131-295.

2. D. Denneberg, Non-additive measure and integral (Kluwer, 1994).

3. G. Greco, Integrale monotone. Rend. Sem. Mat. Univ. Padova 57 (1977), 149-166. 1971)

4. W.A.J. Luxemburg and A.C. Zaanen, Riesz spaces I (North Holland, Amsterdam,

5. J.M. Parker, A note on comonotonic additivity, Glasgow Math. J. 38 (1996), 199-205.

6. D. Schmeidler, Integral representation without additivity. Proc. Amer. Math. Soc. 97 (1986), 255-261.

7. H.J. Skala, On the characterization of certain similarly ordered super-additive functionals, Proc. Amer. Math. Soc. 126 (1998), 1349-1353. 